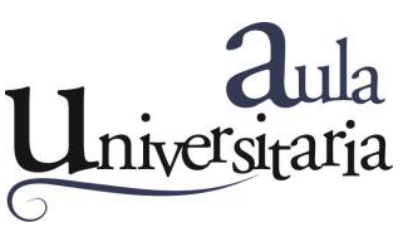

\title{
Una experiencia de comunicación en medicina veterinaria
}

CADOCHE, Lilian S.

\section{Filiaciones institucionales}

Facultad de Ciencias Veterinarias, Universidad Nacional del Litoral.

\section{Correspondencia}

P. Kreder 2805 (3080) Esperanza, Santa Fe. Argentina. Tel. 54-3496-420639.

\section{Resumen}

La correcta comunicación en el mundo actual es imprescindible. El hombre es un ser social, que interactúa cotidianamente con otras personas y, si desea ser un profesional que se inserte adecuadamente en el mercado laboral, no puedo carecer de esta competencia. En este trabajo se intentó recabar información de los alumnos de Medicina Veterinaria de la Universidad Nacional del Litoral, antes y después de dos talleres en los que se trabajó el tema de la comunicación. La encuesta que se empleó permitió ver que, si se trabaja con los alumnos sobre el tema, estos reconocen su importancia y desarrollan habilidades para la interacción y el diálogo tanto con sus compañeros como con el profesor.

\section{Palabras clave}

experiencia | comunicación | taller | veterinaria

\section{Summary}

The correct communication in the current world is essential. The man is a social being, who interacts daily with other people and if he wants to be a professional that fits properly in the labor market he cannot lack this competence. In this work, we attempt to gather information from the students of Veterinary Medicine of the National University of the Litoral, before and after 2 workshops in which the subject of communication was worked on. The survey that was used allowed to see that if the students work on the subject, they recognize its importance and develop skills for interaction, and dialogue with both their peers and the teacher. 


\section{Keywords}

experience / communication / workshop / veterinary

\section{Introducción}

La comunicación es una de las actividades más antiguas de interrelación entre los seres humanos, la cual permite vincularse con otros mediante códigos que consiguen un entendimiento y mantener una retroalimentación cumpliendo el proceso de transmisión de información. Al concebir la comunicación como un proceso y proporcionar importancia a cada uno de sus elementos es más fácil lograr que el objetivo de la misma se cumpla de manera efectiva (Novoa Campos, 2010).

Una primera aproximación a la definición de comunicación puede realizarse a partir de su etimología. La palabra comunicación proviene del latín comunicare, que significa "compartir algo, poner en común algo con otro». Tiene la misma raíz de comunidad, de comunión, y expresa algo que se comparte, que se tiene o se vive en común. Por lo tanto, la comunicación es un fenómeno inherente a la relación que las personas mantienen cuando se encuentran en grupo. A través de ella obtienen información respecto de su entorno y pueden compartirla con el resto.

Por su parte, para Fonseca, comunicar es:

\section{llegar a compartir algo de nosotros mismos. Es una cualidad racional y emocional específica del hombre que surge de la necesidad de ponerse en contacto con los demás, intercambiando ideas que adquieren sentido o significación de acuerdo con experiencias previas comunes. (2000)}

En este sentido, la comunicación es «la transmisión verbal o no verbal de información entre alguien que quiere expresar una idea y quien espera captarla o se espera que la capte» (Etzel y Walker, 2007) mientras que, para Lamb, Hair y McDaniel (2014), la comunicación es «el proceso por el cual intercambiamos o compartimos significados mediante un conjunto común de símbolos».

Cuando nos referimos a que una persona tiene habilidad para la comunicación, decimos que es capaz de: escuchar con atención, no acaparar la palabra, evitar interrumpir, utilizar un lenguaje propio y moderado, lo cual demuestra educación y trato delicado hacia las personas. Algunos de los elementos fundamentales para lograr una buena comunicación son: mostrar interés por la persona, saber preguntar y aprender a escuchar.

Por su parte, la competencia comunicativa es el conjunto de habilidades que posibilita la participación apropiada en situaciones comunicativas específicas. Es la capacidad de escuchar, hacer preguntas, expresar conceptos e ideas de forma efectiva, exponer aspectos positivos, saber cuándo y a quién preguntar para 
llevar adelante un propósito, entre otras. Sin embargo, Avendaño y Perrone (2012), dicen que aun así enfrentamos desacuerdos y discusiones sin sentido, provocando -en ocasiones - una ruptura en las relaciones con los demás. Entender y hacerse comprender son un arte que facilita la convivencia y la armonía en todo lugar.

Se puede agregar a lo expresado el resultado de un estudio que sobre comunicación se realizó en la Universidad de California, en el cual se expuso en orden de importancia el porcentaje de los diferentes factores de la comunicación: el lenguaje corporal, el tono de voz y las palabras (Guix, 2011).

Creemos que la conceptualización que realizan Hernández Mendo y Garay (2005) expresa claramente lo que para nuestro caso queremos estudiar: la comunicación es un proceso de interacción social de carácter verbal o no verbal, con intencionalidad de transmisión y que puede influir, con y sin intención, en el comportamiento de las personas que están en la cobertura de dicha emisión.

En síntesis, la comunicación es indispensable para procurar y mantener las buenas relaciones en todos los ámbitos de nuestra vida, particularmente en la familia, el trabajo e indudablemente para cualquier profesional cuya tarea se centre en la interacción social. El médico veterinario especialmente necesita de esta competencia de manera imperativa e inexcusable

Pero para empoderar al joven estudiante y futuro profesional de esta habilidad también necesitamos analizar la comunicación educativa, pues es a través de ella que se puede desarrollar y fortalecer la propia. $Y$ cuando «hablamos de comunicación educativa, hablamos de aquella que se produce en un contexto especial -el ámbito educativo - y sin la cual la posibilidad de educar es imposible» (Charles, 1991).

Para analizar el proceso comunicacional tomaremos como referencia el modelo comunicativo diseñado por Berlo (1984), que establece como actores en el proceso de la comunicación educativa al docente y al discente. Según este modelo, los elementos involucrados son los contenidos educativos, el soporte educativo, los medios didácticos, los procesos de codificación y decodificación y el de realimentación.

La comunicación educativa establecida por este mecanismo tiene como fin último el intercambio recíproco de conocimientos, procedimientos y actitudes que constituyen, en definitiva, la finalidad de la educación. Por ello, Murga Menoyo (2012) dice que esta comunicación debe tener ciertos requisitos funcionales y que, para que sea eficiente, debe ser:

- Motivadora: el docente tiene que transmitir en la comunicación educativa estímulos que permitan captar y mantener la atención del alumno ya sea por la creación de una necesidad o por la satisfacción de ella.

- Persuasiva: debe lograr su finalidad en función a los objetivos o a las metas. 
- Estructurante: la comunicación debe ser capaz de orientar, facilitar y promover la construcción de conocimientos del educando y ayudar no solo a identificar la estructura jerárquica en la que se inscribe el mismo, sino también su relación con los organizadores previos de la estructura cognoscitiva de los discentes.

- Adaptativa: esta característica de la comunicación educativa es la que tiende a facilitar la interacción del discente con el medio en el que este se desenvuelve a través del mejoramiento del repertorio de conductas expresivas del sujeto.

- Generalizadora: la comunicación educativa debe tener capacidad para inducir a nuevas interconexiones o relaciones que permitan la aplicación del conocimiento adquirido a circunstancias análogas.

- Facilitadora de la inteligibilidad: esta meta se consigue al «adaptar la información a las circunstancias que posibilitan su captación por el sujeto». Esto supone un cuidadoso análisis de los canales, los medios de reforzamiento de mensajes, los ritmos y la progresión, entre otros.

Para algunos autores, como Sarramona (1988), educar es comunicar, y:

comunicar significa dialogar lo que da lugar a una forma particular de relacionarse, de establecer un intercambio que genera reflexión, crítica y construcción de significados compartidos, a través de una negociación constante entre los participantes. Implica horizontalidad e interacción.

Ojalvo (2000) la define como:

la comunicación del maestro con los escolares en el proceso de enseñanza, que crea las mejores condiciones para desarrollar la motivación del alumno y el carácter creador de la actividad docente, para formar correctamente la personalidad del alumno.

La comunicación educativa es un proceso de interacción entre profesores, estudiantes y estos entre sí y de la escuela con la comunidad, que tiene como finalidad crear un clima psicológico favorable para optimizar el intercambio y recreación de significados que contribuyan al desarrollo de la personalidad de los participantes.

Entonces, el proceso educativo con nuevas características trae consigo una forma diferente de comunicación que va más allá de la mera transmisión de información, forma parte de un proceso de interacción e intercambio entre el 
docente y los estudiantes. En este marco, el docente se convierte en un mediador pedagógico en el momento en que su trabajo no es mecánico, sino que se interesa porque los estudiantes asimilen nuevos conocimientos y está pendiente de las debilidades, fortalezas y necesidades de los alumnos en el proceso educativo.

La mayor parte de las actividades que acontecen en el mundo académico y laboral lo hace en escenarios y situaciones de naturaleza interpersonal. Por ello, la comunicación es la habilidad de gestión más importante sin la cual otras habilidades o competencias no resultarían operativas. Las posibilidades de trabajo, estudio, relaciones sociales y superación dependen, en buena parte, de nuestra capacidad para interactuar con los demás teniendo a la expresión oral como herramienta fundamental. Es necesario, por tanto, que sea una habilidad clave para la formación universitaria ya que la comunicación como cualquier otra competencia se puede desarrollar y mejorar desde el aula.

En particular, se hace más evidente un creciente reconocimiento en la veterinaria clínica de la importancia de este campo para un desarrollo profesional adecuado (Hafen et al., 2009). Varios estudios realizados parecen coincidir en sus resultados y dejan ver que, mientras las habilidades técnicas y científicas de la profesión veterinaria permanecen a un buen nivel, hay evidencia de que los veterinarios poseen escasas habilidades comunicativas y administrativas (Brown y Silverman, 1999).

Según Blanco (2009), el desarrollo de las competencias comunicativas en general y de la competencia comunicativa oral en especial, en los estudiantes terciarios y universitarios, implica la adquisición práctica de determinadas capacidades, habilidades, destrezas y actitudes que no tienen nada que ver con la adquisición y transmisión de conocimientos y menos con el tradicional dictado de clases del docente. La mejor manera de desarrollar estas habilidades es participando en situaciones comunicativas reales, en las que se trata de simular situaciones de la vida real. Esto confiere un carácter práctico y dinámico aumentando el atractivo y el interés de los alumnos por la materia, en definitiva, su motivación por aprender.

Nuestra propuesta se cimenta en estas consideraciones, debemos, podemos y deseamos no descuidar en la formación profesional el desarrollo y ponderación de las competencias de comunicación de nuestros futuros médicos veterinarios. Para conocer y ayudar a los alumnos a mejorar sus habilidades para la comunicación decidimos organizar talleres sobre este tema, en los que se intentó dar a conocer a los estudiantes los elementos fundamentales de una correcta comunicación. Se les brindó un material teórico sobre el tema y se les mostraron videos entretenidos para llamar su atención sobre la importancia de una correcta comunicación. 


\section{Objetivo}

El objetivo que se propuso en este trabajo fue analizar si hubo cambios en las autopercepciones de los alumnos que participaron de los talleres respecto de su habilidad para comunicarse. La hipótesis inicial, que sostuvimos es que la habilidad de comunicación no es un talento natural, sino algo que puede ser aprendido y desarrollado si se lo estimula para ello. Ese fue el motivo que nos estimuló para desarrollar los talleres que mencionamos. De ellos recogimos información respecto de la percepción que de sí mismo tenían los alumnos sobres sus habilidades para la interacción social y la comunicación.

\section{Metodología}

Para la recolección de la información que nos permitiera conocer si los alumnos habían mejorado sus habilidades para la comunicación y las habilidades sociales necesarias para una correcta interacción empleamos un cuestionario de 28 preguntas que fue puesto en el google drive para que los participantes de estos encuentros los pudieran responder en sus casas, a posteriori de su participación en los talleres.

Participó de estos talleres un número importante de 60 alumnos de distintos niveles de la carrera. Los mismos tuvieron una duración de 4 horas, en las dos primeras se dieron lineamientos generales sobre los conceptos involucrados en una correcta comunicación tanto coloquial como académica, y en las dos horas posteriores se realizaron tareas de interacción entre los alumnos que se organizaron en grupos y debían responder a consignas en las que se les preguntaba si entendieron lo que se dijo al comienzo del encuentro y qué aportes recibieron de los mismos. Al finalizar cada taller, se les pidió que respondieran los cuestionarios que nos permitieron evaluar la efectividad del intento educativo. Las preguntas tenían como opciones: no nunca o casi nunca; normalmente no; a veces; si, siempre o casi siempre

Algunas de las preguntas que formaron parte del cuestionario fueron:

1. ¿Cuándo me encuentro con alguien por primera vez trato de dar una buena impresión?

2. ¿Cuándo hablo lo hago oportunamente y me expreso de forma correcta?

3. ¿Me encuentro animado al hablar con gente conocida? ¿Mi lenguaje corporal es rico?

4. ¿Empleo la modulación el volumen de voz para reforzar lo que digo? 
5. ¿Cuándo participo de una conversación trato de ser amable?

6. ¿Mis habilidades de comunicación me han abierto muchas puertas?

7. ¿Comprendo la importancia del dominio de mí mismo y lo practico?

8. ¿Interactúo bien con la gente porque entiendo todos los elementos implicados en la conversación?

9. ¿Cuido lo que digo porque sé que mis opiniones siempre tienen que ser escuchadas por todos?

10. ¿Puedo permanecer en silencio a propósito pues ejerzo un buen control sobre cada palabra que pronuncio?

11. ¿Cuándo entablo un diálogo trato de ser prudente en las palabras que empleo?

12. ¿Creo que las indiscreciones en un equipo de trabajo son graves y negativas?

13. ¿Participo en altercados o discusiones?

14. ¿Siento que logro comunicarme bien cuando entablo una conversación?

15. ¿Sé que en ocasiones es mejor guardar silencio?

16. ¿Empleo palabras malsonantes en un ambiente social o laboral?

17. ¿Sé escuchar a los demás y presto atención a lo que me dicen?

18. ¿Sé cuándo debo dejar hablar a los demás?

19. ¿Soy eficaz cuando se trata de persuadir a las personas de que comprendan mi punto de vista?

20. ¿A pesar de que me gustaría ser franco creo que a veces no es necesario decir la verdad? 


\section{Resultados}

Del análisis que realizamos en las encuestas en el primer y segundo taller, los participantes respondieron a algunas de las consignas de la encuesta de la siguiente manera:

A la consigna «¿Cuándo hablo lo hago oportunamente y me expreso de forma correcta?» un $13 \%$ respondió en la primera encuesta que "sí, siempre o casi siempre» y un $87 \%$ «a veces». Pero afortunadamente cuando terminaron los dos talleres este porcentaje aumentó a un 17,4 \% que respondió «sí, siempre o casi siempre», y un $82,6 \%$ «a veces».

Cuando en el primer taller se les preguntó si empleaban bien la modulación y el volumen de voz para reforzar lo que decían, el 8,7 \% de los encuestados contestó que «no»; un 43,5 \% dijo «a veces»; un 17,4\%, «normalmente no»; y un 30,4\% expresó «sí, siempre o casi siempre». Al finalizar el segundo taller estos porcentajes aumentaron, ya que un $6 \%$ afirmó que «no»; un $36 \%$ afirmó que «a veces»; un $15 \%$, «normalmente no»; y un $43 \%$, «sí, siempre o casi siempre».

A la consigna «¿Mis habilidades me han abierto muchas puertas?», en el primer encuentro respondió «sí, siempre o casi siempre» un 34,8 \%; un 8,7 \%, «no»; un $13 \%$, «normalmente no»; y un 43,5\%, «a veces». En el segundo taller, estos porcentajes se modificaron, ya que un $36 \%$ respondió «sí, siempre o casi siempre»; un $6 \%$, «no»; un $10 \%$, «normalmente no»; y un $48 \%$, «a veces».

A la pregunta « ¿Interactúo bien con la gente porque entiendo todos los elementos implicados en la comunicación?», en el primer taller, los alumnos contestaron un 36,4\% «sí»y un 63,6 \% a veces, mientras que en el segundo encuentro estos porcentajes se modificaron, ya que un $45 \%$ dijo «sí » y un $55 \%$ «a veces».

A la consigna «¿Cuándo entablo un diálogo trato de ser prudente en las palabras que empleo?», en el primer taller un 47,6 \% respondió «sí, casi siempre, siempre»; un 47,6 \%, «a veces»; y un 4,8\%, «normalmente no». Cuando se los consultó en el segundo taller los porcentajes fueron un $52 \%$ «sí»; un $32 \%$ expresó «a veces»; y un $16 \%$, «normalmente no».

Cuando se les preguntó «¿Sé escuchar a los demás y presto atención a lo que me dicen?», los porcentajes fueron, para el primer taller, un 61,9 \% que «sí »; un $9,5 \%$ «normalmente no»; y un $28,6 \%$ «a veces». En el segundo encuentro, estos porcentajes cambiaron, de tal manera que un $70 \%$ dijo «sí»; un $5 \%$ expresó «normalmente no»; y un $25 \%$, «a veces».

En el primer encuentro, ante la pregunta « ¿Sé cuándo debo dejar hablar a los demás?», el 57,1 \% respondió «síl» y un 42,9\%, «a veces». Mientras que en el segundo taller un $65 \%$ expresó «sí» y un $35 \%$, «a veces».

En el primer encuentro, ante la consigna « ¿Soy eficaz cuando se trata de persuadir a las personas que comprendan mi punto de vista», un 28,6 \% dijo «sí»; un 14,3\%, «normalmente no»; y un 51,1\%, «a veces». Es interesante 
observar que en el segundo taller estos porcentajes aumentaron, de modo tal que un $45 \%$ expresó «sí»; un $12 \%$, «normalmente no»; y un $43 \%$, «a veces».

\section{Conclusiones}

Del análisis que realizamos en la primera encuesta a los alumnos participantes de los talleres, podemos ver que al informarlos respecto de qué es la comunicación, conceptualizándola y mostrándoles videos divertidos que mostraran los beneficios de una correcta comunicación, los alumnos se mostraron muy interesados, participaron activamente de las tareas, intercambiaron opiniones, solicitaron más material y que se realicen nuevos encuentros para debatir acerca de estos temas. El mercado laboral exige, al mismo nivel que los conocimientos técnico-científicos, la posesión de habilidades sociales, entre las cuales la comunicación ocupa el primer lugar. 


\section{Referencias bibliográficas}

- Blanco, A. (Ed.) (2009). Desarrollo y Evaluación de competencias. En Educación Superior. La comunicación oral y la presentación eficaz de ideas. Narcea.

- $\quad$ Brown, J.P. y Silverman, J.D. (1999). The current and future market for veterinarians and veterinary medical services in the United States. Journal of the American Veterinary Medical Association, 215(2), 161-183.

- Charles, M. (1991). Comunicación y procesos educativos. Tecnología y Comunicación Educativa, (17), 17-23.

- Avendaño, F. y Perrone, A. (2012). El aula: un espacio para aprender a decir y a escuchar: estrategias y recursos. Homo sapiens.

- Berlo, D. (1984). El proceso de la comunicación. 14ta. ed. Ateneo.

- $\quad$ Etzel, M.; Walker, B. (2007). Fundamentos de Marketing. 14ta. ed. McGraw-Hill Interamericana.

- Fonseca, M. (2000). Comunicación Oral Fundamentos y Práctica Estratégica. Pearson Educación.

- Guix, X. (2011). Ni me explico, ni me entiendes. Laberintos de la comunicación. 10ma. ed. Urano.

- Hafen, M.; Rush, B.R. y Nelson, S.C. (2009). Utilizing filmed authentic student client interactions as a communication teaching tool. Journal of Veterinary Medical Education, (36), 429-435.

- Hernández Mendo, A. y Garay, O. (2005). La comunicación en el contexto deportivo. En Hernández Mendo, A. (Coord.). Psicología del deporte. Vol. 1. Wanceulen Editorial Deportiva.

- Lamb, C.; Hair, J.; y McDaniel, C. (2014). Marketing. 11ra. ed. Ediciones Paraninfo.

- Murga Menoyo, M.A. (2012). La Docencia en la Enseñanza y Aprendizaje Abiertos y a Distancia. Master Internacional en Enseñanza y Aprendizaje Abiertos y a Distancia. Teorías de la Comunicación y Enseñanza a Distancia. Máster en EAAD. Universidad Nacional de Educación a Distancia, España.

- Novoa Campos, G. (2010). Lecciones de oratoria. Pluma y papel.

- Ojalvo, V. (1994). La comunicación en el aula. Revista Cubana de Educación Superior, $4(1), 29-34$.

- Ojalvo, V. (2000). Competencia Comunicativa y educación de los valores en estudiantes universitarios. Universidad 2000.

- Sarramona, J. (1988). Comunicación y Educación. Ediciones CEAC. 